\title{
Grid Intersection and Box Intersection Graphs on Surfaces (Extended Abstract)
}

\author{
Jan Kratochvíl ${ }^{1 \star}$ and Teresa Przytycka ${ }^{2}$ \\ 1 Charles University, Prague, Czech Republic \\ 2 Odense University, Odense, Denmark
}

\begin{abstract}
As analogs to grid intersection graphs and rectangle intersection graphs in the plane, we consider grid intersection graphs, grid contact graphs and box intersection graphs on the other two euclidean surfaces - the annulus and the torus. Our first results concern the inclusions among these classes, and the main result is negative - there are bipartite box intersection graphs on annulus (torus), which are not grid intersection graphs on the particular surfaces (in contrast to the planar case, where the two classes are equal, cf. Bellantoni, Hartman, Przytycka, Whitesides: Grid intersection graphs and boxicity, Discrete Math. 114 (1993), 41-49). We also consider the question of computational complexity of recognizing these classes. Among other results, we show that recognition of grid intersection graphs on annulus and torus are both polynomial time solvable, provided orderings of both vertical and horizontal segments are specified.
\end{abstract}

\section{Motivation}

Intersection graphs of different types of geometric objects in the plane gained a lot of attention in the past years. One may consider interval graphs, circle graphs, circular arc graphs, permutation graphs and string graphs, to mention just a few of such classes of graphs. At this point, we want to pay closer attention to grid intersection graphs (intersection graphs of vertical and horizontal straight line segments in the plane, such that no two parallel segments of the representation overlap cf. [1], [4]), grid contact graphs (grid intersection graphs that have a representation in which no two segments cross each other cf. [3]) and box intersection graphs (graphs of boxicity two, i.e. intersection graphs of isothetic rectangles in the plane cf. [7], [10], [4], [1]).

The concept of vertical and horizontal directions translates naturally to the other two orientable euclidean surfaces - the annulus and the torus, and our aim

\footnotetext{
* This research was started when the first author was visiting Odense University in May 1994. The paper was written when the first author visited University of Oregon in Eugene, Oregon in the academic year 1994/5. The first author also acknowledges partial support from Czech Research Grants GAUK 361 and GAČR 2167.
} 
is to study analogous classes of graphs on these surfaces. Our motivation stems from two results about the planar case, whose generalizations we question.

First, Bellantoni et al. [1] proved that every bipartite graph of boxicity two is in fact a grid intersection graph. In other words, given a set of rectangles in the plane such that its intersection graph is bipartite, one can shrink the rectangles corresponding to the vertices of one color class into vertical segments, and the rectangles corresponding to the other color class into horizontal segments, while keeping the intersection graph itself unchanged.

The second source of our motivation is the result of de Frayssiex et al. [3], which says that every planar bipartite graph is a grid contact graph. This result, which is based on visibility representations of general planar graphs $[8,9]$, was actually generalized to the torus by Mohar and Rosenstiehl [6].

The paper is organized as follows. In Section 2, we give exact definitions of the classes of graphs under consideration. Then, in Section 3, we present the inclusions among the classes (and reason about their strictness). In Section 4, we aim at the question of the computational complexity of recognizing the considered classes of graphs, in particular in the case when the coordinates of the segments are preordered. The last section contains final remarks and open problems.

\section{Definitions}

It is usual to view the torus as a rectangle whose vertical and horizontal sides are unified. In this model, segments of horizontal and vertical lines correspond to segments of geodetic circles on the torus. Having this model in mind, we will work with a rectangle whose vertical sides are unified as a model of the annulus, and with ordinary rectangle as a model of the plane. (Since we will only consider finite graphs, restriction to bounded parts of annulus and plane are irrelevant.) We will refer to this rectangle as the base rectangle. We will use the abreviations $p l$, an and to for the plane, the annulus and the torus, respectively. For each of these three surfaces, we consider the following classes of graphs (note that in view of the theorems whose generalizations we question, we only consider bipartite graphs):

GI(surface) $=$ grid intersection graphs on the surface $=$ graphs that have a representation by vertical and horizontal line segments on the surface, such that any two parallel segments are disjoint and two segments share a point if and only if the corresponding vertices are adjacent;

$\mathrm{GC}($ surface $)=$ grid contact graphs on the surface = graphs which have a representation by vertical and horizontal line segments on the surface, such that any two parallel segments are disjoint, no two segments cross and two segments share a contact point if and only if the corresponding vertices are adjacent;

$\mathrm{BI}($ surface $)=$ box interesection graphs on the surface = bipartite graphs that have a representation by isothetic rectangles on the surface such that two rectangles are disjoint if and only if the corresponding vertices are nonadjacent; 
$\mathrm{EM}($ surface $)=$ graphs embeddable in the surface $=$ bipartite graphs that allow a noncrossing drawing on the surface.

As an illustrative example, a grid contact representation of $K_{4,4}$ on the torus is shown in Figure 1.

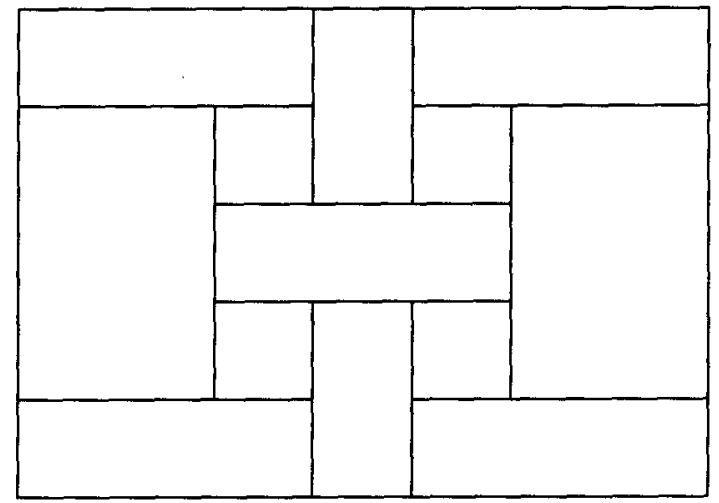

Fig. 1. A grid contact representation of $K_{4,4}$ on the torus.

We will view rectangles (boxes) as two-dimensional intervals, i.e., as products of intervals in the coordinates. In the case of the torus, both horizontal and vertical intervals that determine a box may be wrapped around the sides of the base rectangle, i.e., the coordinate of the left endpoint may be greater than the coordinate of the right one. In the case of the annulus, only the horizontal intervals may be of this type, and in the planar case, all intervals must be proper. For the case of grid intersection and grid contact graphs, the segments will be viewed as boxes with one side of length zero. Since we only deal with finite graphs, we may assume without loss of generality that the coordinates of the endpoints of the intervals that determine the boxes of a representation are mutually distinct integers.

It is well known that a graph is a box intersection graph in the plane if and only if it is the intersection of two interval graphs, i.e., if it has interval dimension at most 2 [2]. Similarly, a graph is a box intersection graph on the annulus if and only if it is the intersection of an interval graph and a circular arc graph. Finally, a graph is a box intersection graph on the torus if and only if it is the intersection of two circular arc graphs, i.e., if it has circular arc dimension at most 2. 


\section{Inclusions among the classes}

The following three propositions are straightforward:

Proposition 1. For any $X \in\{E M, G I, G C, B I\}, X(p l) \subseteq X(a n) \subseteq X(t o)$.

Proposition 2. For any $X \in\{p l$, an, to $\}, G C(X) \subseteq G I(X) \subseteq B I(X)$.

Proposition 3. For any $X \in\{p l, a n, t o\}, G C(X) \subseteq E M(X)$.

The last proposition is in fact a special case of a more general theorem which says that a contact graph of curves on any surface with at most two curves sharing a contact point is embeddable on that surface.

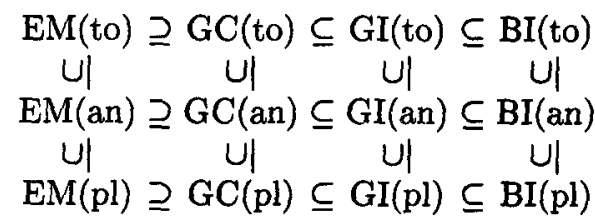

Fig. 2. Inclusions among the classes.

All these inclusions are depicted in Figure 2. It follows from the results of Bellantoni et al. [1] that $\mathrm{GI}(\mathrm{pl})=\mathrm{BI}(\mathrm{pl})$. Another inclusion in the bottom line reduces to equality due to the result of de Frayssiex et al. [3], namely $\operatorname{EM}(\mathrm{pl})=$ $\mathrm{GC}(\mathrm{pl})$. But every graph embeddable on annulus is planar (the cylinder is topologically equivalent to the plane with two points removed), and hence EM(pl) $=\mathrm{EM}(\mathrm{an})=\mathrm{GC}(\mathrm{an})=\mathrm{GC}(\mathrm{pl})$. It is well known that e.g. $K_{3,3}$ is embeddable on torus and not in the plane, hence $\operatorname{EM}(a n) \subset \operatorname{EM}($ to $)$. Concerning the other inclusions, we have the following theorem:

Theorem 4. All other inclusions are strict, as depicted in Figure 3.

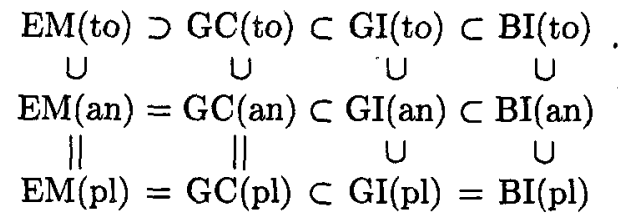

Fig. 3. Inclusions among the classes. 
Let us remark at this point that the equality $\mathrm{EM}($ to $)=\mathrm{GC}($ to $)$ "almost" holds - Mohar and Rosenstiehl proved that every bipartite graph embeddable on the torus is indeed a grid contact graph on a skew torus (i.e., a torus whose horizontal and vertical grid directions are not parallel with the sides of the base rectangle). However, there are graphs embeddable on the torus that are not grid contact graphs on the torus in our sense [5]. The strict inclusion GC(an) $\subset$ $\mathrm{GC}$ (to) follows from the complete bipartite graph $K_{3,3}$, the inclusions $\mathrm{GC}(X)$ $\subset \mathrm{GI}(X), X \in\{$ pl,an,to $\}$ follow from complete bipartite graphs as well. For the remaining inclusions, we use slightly more involved constructions of graphs that have topologically unique intersection representations. We will give the corresponding constructions in the full version of the paper.

\section{Recognition of the classes}

The first author has proved in [4] that recognition of grid intersection graphs in the plane (and hence also of graphs of boxicity 2) is NP-complete. By a straightforward reduction we can show the following theorem.

Theorem 5. Recognition of grid intersection and box intersection graphs on the annulus and torus are NP-complete problems.

Sketch of the proof. Let $G$ be a graph whose membership to GI(pl) we want to test. Take a nonplanar graph, say $H$, embeddable on the torus and let $H^{*}$ be the graph obtained from $H$ by subdividing its edges by sufficiently many vertices. Then $H^{*}$ can be represented as a grid intersection graph on the torus, but every face of any such representation is homeomorphic to a disk. Hence $H^{*}+G$, the disjoint union of $H^{*}$ and $G$, is in GI(to) if and only if $G \in \mathrm{GI}(\mathrm{pl})$.

A slightly more involved construction works for the annulus. Here we start with a 3 -connected planar graph $H$ which has at least 3 faces. Again, we take the subdivided graph $H^{*}$. To obtain a graph $G^{\prime}$, we place a copy of $G$ in each of three chosen faces of $H^{*}$, connected to the vertices of the faces by paths of suitable length. In any grid intersection representation of $H^{*}$ on the annulus, at least one of the three chosen faces bounds a region homeomorphic to a disk. Hence $G^{\prime} \in \mathrm{GI}(\mathrm{an})$ if and only if $G \in \mathrm{GI}(\mathrm{pl})$.

Other interesting questions arise if we consider grid intersection graphs with preordered color classes. To be precise, we consider the following problem: Let $G$ be a bipartite graph with color classes $V$ and $H$ and let the vertices be ordered $V=\left\{v_{1}, v_{2}, \ldots, v_{n}\right\}, H=\left\{h_{1}, h_{2}, \ldots, h_{m}\right\}$. We want to find a grid intersection representation for $G$ in which each vertex $v_{i}$ will be represented by a vertical segment with endpoint coordinates $\left[i, b_{i}\right],\left[i, u_{i}\right]$, and each vertex $h_{j}$ will be represented by a horizontal segment with endpoints $\left[l_{j}, j\right],\left[r_{j}, j\right]$. (If $b_{i}<u_{i}$ then the segment is proper, otherwise it wraps around the horizontal sides of the base rectangle. This is allowed only in the case of the torus. Similarly, if $l_{j}>r_{j}$, then the corresponding horizontal segment wraps around the vertical sides of the base rectangle. This is allowed both in the case of the annulus and the 
torus.) In the planar case, there is a very straightforward way to decide if such an assignment exists. For each "horizontal" vertex $h_{j}$, obviously $l_{j}$ must be $\leq$ $\min \left\{i \mid v_{i} h_{j} \in E(G)\right\}$ and $r_{j}$ must be $\geq \max \left\{i \mid v_{i} h_{j} \in E(G)\right\}$. Hence if an assignment exists, then assigning $l_{j}=\min \left\{i \mid v_{i} h_{j} \in E(G)\right\}, r_{j}=\max \left\{i \mid v_{i} h_{j} \in E(G)\right\}$, $b_{i}=\min \left\{j \mid v_{i} h_{j} \in E(G)\right\}, u_{i}=\max \left\{j \mid v_{i} h_{j} \in E(G)\right\}$ yields an intersection representation, which is if and only if there are no indices $i_{1}<i<i_{2}, j_{1}<j<j_{2}$ such that $v_{i_{1}} h_{j}, v_{i_{2}} h_{j}, v_{i} h_{j_{1}}, v_{i} h_{j_{2}}$ are edges of $G$ and $v_{i} h_{j}$ is not. This condition is actually much better seen from the bipartite adjacency matrix of $G$. This matrix $A_{G}$ is a matrix of type $n \times m$, and $\left(A_{G}\right)_{i j}=1$ if $v_{i} h_{j} \in E(G)$ and $\left(A_{G}\right)_{i j}=0$ otherwise. The necessary and sufficient condition for the existence of ordered grid intersection representation descirebed above is restated as "the bipartite adjacency matrix $A_{G}$ does not contain a submatrix of the following form:"

$$
\left(\begin{array}{ccc}
1 \\
1 & 0 & 1 \\
1
\end{array}\right)
$$

This description straightforwardly yields a polynomial recognition algorithm for ordered grid intersection graphs in the plane.

The situation is more interesting for grid intersection graphs on the annulus and the torus. Here we have:

Theorem 6. A bipartite graph with preordered color classes has an ordered grid intersection representation on the annulus if and only if its bipartite adjacency matrix does not contain a submatrix of the following type

$$
\left(\begin{array}{cccc} 
& & & 1 \\
& 1 & & \\
1 & 0 & 1 & 0 \\
& 1 & & \\
& & & 1
\end{array}\right)
$$

(where the first two and/or the last two rows may be swapped or may coincide). Consequently, there is a polynomial algorithm that decides if a preordered bipartite graph has an ordered grid intersection representation on the annulus.

Obviously, a finite number of forbidden submatrices can be read out from the pattern given in the theorem. On the other hand, we can prove that in the case of ordered grid intersection representations on the torus, the number of minimal forbidden submatrices is infinite. Despite of this fact, we can prove:

Theorem 7. There is a polynomial algorithm that decides if a given bipartite preordered graph has an ordered grid intersection representation on the torus.

Proof. Given a preordered bipartite graph $G=(V \cup H, E)$, we will consider its adjacency matrix $A$. Every row (and column) of the matrix consists of continuous intervals of zeros separated by intervals of ones. Let $I_{i, 1}, I_{i, 2}, \ldots, I_{i, n_{i}}$ (resp. $\left.Y_{j, 1}, Y_{j, 2}, \ldots, Y_{j, m_{j}}\right)$ be the continuous intervals of coordinates of zeros in the $i$-th 
row (resp. $j$-th column). (Note that again, one of these intervals may be nontrivial, i.e. may have the left endpoint placed to the right of the right endpoint.) We say that the horizontal segment $\overline{\left[l_{i}, i\right]\left[r_{i}, i\right]}$ representing a vertex $h_{i}$ misses an interval $I_{i, j}$ if $I_{i, j} \cap\left[l_{i}, r_{i}\right]=\emptyset$, and we say that this segment covers $I_{i, j}$ if $I_{i, j} \subset\left[l_{i}, r_{i}\right]$. The analogous notions for vertical segments and column-intervals are defined in obvious way.

Note that a general grid intersection representation may contain segments that neither miss nor cover a particular interval of zeros. However, it is easy to argue that if $G$ has an ordered grid intersection representation, then it has a representation whose every horizontal (vertical) segment misses exactly one interval of zeros in the particular row (resp. column) and covers all the others. Such special representations can be easily described as instances of 2-SATISFIABILITY, a problem which is notoriously known to be solvable in polynomial time.

For every interval $I_{i, j}$, we introduce a variable $x_{i, j}$, and for every interval $Y_{r, s}$, a variable $y_{r, s}$. The idea is to construct a formula which would be satisfied if and only if $G$ has an ordered grid intersection representation, under a truth valuation which encodes $x_{i, j}=$ true iff the segment representing $h_{i}$ misses the interval $I_{i, j}$ (resp. $y_{r, s}=$ true iff the segment representing $v_{r}$ misses the interval $Y_{r, s}$ ). The requirement that the segment representing $h_{i}$ misses at most one interval is then expressed by a subformula

$$
\Phi_{i}=\bigwedge_{1 \leq j<k \leq n_{i}}\left(\neg x_{i, j} \vee \neg x_{i, k}\right) .
$$

The analogous requirement for vertical segment $v_{r}$ is expressed by

$$
\Psi_{r}=\bigwedge_{1 \leq s<t \leq m_{r}}\left(\neg y_{r, s} \vee \neg y_{r, t}\right) .
$$

Any truth assignment that satisfies all these subformulas will correspond to a grid intersection representation which will realize all necessary crossings of the segments (i.e., all ones of the adjacency matrix). To avoid undesirable crossings, we have to ensure that for every zero in the adjacency matrix, at least one of the two intervals (vertical and horizontal) is missed by the coresponding segments of the representation. For a particular position $i, r$ such that $A_{i r}=0, r \in I_{i, j}$ and $i \in Y_{r, s}$, this requirement is expressed by a subformula

$$
\Phi_{i, r}=x_{i, j} \vee y_{r, s}
$$

It is now clear that $G$ has an ordered grid intersection representation if and only if the following formula $\Phi$ is satisfiable

$$
\Phi=\bigwedge_{i=1}^{n} \Phi_{i} \wedge \bigwedge_{r=1}^{m} \Psi_{r} \wedge \bigwedge_{i, r: A_{i r}=0} \Phi_{i, r}
$$

All clauses that appear in $\Phi$ have size 2 and hence the ordered grid intersection representability problem is reduced to 2-SATISFIABILITY. 


\section{Concluding remarks}

The computational complexity of the ordered grid intersection problem is open if only one of the color classes comes preordered (and the vertices in the other color class can be permuted). For the planar case, this problem (which was first considered by the first author and J. Nešetril) appears in [4]. If one does not believe in existence of a polynomial solution to this problem, then the analogous problems for annulus and torus should be easier to be proved NP-complete.

In the view of the nonequivalence of $\mathrm{EM}(\mathrm{to})$ and $\mathrm{GC}(\mathrm{to})$ [5], it would be very interesting to know a description of the class of grid contact graphs on the torus. In particular, is this class recognizable in polynomial time?

\section{References}

1. Bellantoni, S., Ben-Arroyo Hartman, I., Przytycka, T., Whitesides, S.: Grid intersection graphs and boxicity, Discrete Math. 114 (1993), 41-49

2. Cozzens, M.B., Roberts, F.S.: On dimensional properties of graphs, Graphs and Combinatorics 5 (1989), 29-46

3. de Fraysseix, H., de Mendez, P.O., Pach, J.: Representation of planar graphs by segments, Intuitive Geometry, Colloquia Mathematica Societatos Janos Bolyai 63 (1991), 109-117

4. Kratochvíl, J.: A special planar satisfiability problem and a consequence of its NPcompleteness, Discrete Appl. Math. 52 (1994) 233-252

5. Mohar, B. (private communication)

6. Mohar, B., Rosenstiehl, P.: Tessellation and visibility representations of maps on the torus (preprint)

7. Roberts, F.S.: On the boxicity and cubicity of a graph, In: W.T. Tutte, ed., Recent Progress in Combinatorics, Academic Press, New York, 1969, pp. 301-310

8. Rosenstiehl, P., Tarjan, R.E.: Rectilinear planar layouts and bipolar orientations of graphs, Discrete Comput. Geometry 1 (1986), 343-353

9. Tamassia, R., Tollis, I.G.: A unified approach to visibility representations of planar graphs, Discrete Comput. Geometry 1 (1986), 321-341

10. Wood, D.: The riches of rectangles, In: Proceedings 5th International Meeting of Young Computer Scientists, Smolenice (1988), 67-75 